\title{
Peculiarities of high electric field conduction in p-type diamond
}

\author{
V. Mortet ${ }^{1,2}$, D. Trémouilles ${ }^{3}$, J. Bulir ${ }^{1}$, P. Hubík ${ }^{1}$, L. Heller $^{1}$, E. Bedel-Pereira ${ }^{3}$, A. Soltani ${ }^{4}$
}

${ }^{1}$ Institute of Physics CAS, v.v.i., Na Slovance 2,182 21, Prague 8, Czech Republic

${ }^{2}$ Czech Technical University in Prague, Faculty of Biomedical Engineering, Sitna 3105, 272 01, Kladno, Czech Republic

${ }^{3}$ LAAS-CNRS, Université de Toulouse, CNRS, Toulouse, France

4 Institut d'Electronique, Microélectronique et Nanotechnologie, UMR-CNRS 8520, Université Lille, Cite Scientifique, Avenue Poincaré, CS 60069, 59652 Villeneuve d'Ascq Cedex, France.

Electrical properties of chemical vapour deposited p-type epitaxial diamond layers are studied in high electric field conditions. Quasi-static current-voltage characteristics have been measured using Transmission-Line Pulse method with 100 ns pulses. Reproducible impurity impact ionization avalanche breakdown occurs at critical electrical field in the range of $100-200 \mathrm{kV} . \mathrm{cm}^{-1}$ depending on acceptor concentration and temperature, leading to complete ionisation of neutral impurities. Current-voltage characteristics exhibit an S-shape with bi-stable conduction characteristic of impurity impact ionisation.

Due to their high ionisation energy, dopants in diamond are only partially ionised at room temperature, e.g. less than $0.1 \%$ of boron impurities are ionised in low-doped p-type diamond. As matter of fact, the temperature range of incomplete ionisation of boron acceptor, i.e. the freeze out region, extends up to about $1000 \mathrm{~K}$. The consequent high resistivity severely limits potential applications of diamond as semiconductor ${ }^{1,2}$. In the freeze out regime, the conductivity can be increased by excitation of free carriers bounded to neutral impurity centres to conduction/valence band by 1 - thermal ionisation rising the temperature, 2- optical ionisation with light irradiation of suitable wavelength and finally 3impact ionization of neutral centres by the free carriers under sufficiently high electric field. Under high electric field, free carriers can gain enough energy to be able to ionised neutral impurities by inelastic collisions leading to free carrier multiplication. This process results in nonlinear current voltage characteristics ${ }^{3}$. Impact ionization of impurities has been already studied in narrow band gap semiconductor like germanium ${ }^{3}$ and silicon $^{4}$ at cryogenic temperature, and it has been very recently reported in wide band gap semiconductor like silicon carbide polytypes ${ }^{5}$ and diamond ${ }^{6}$ at room temperature. 
In this paper, we present further experimental data on reversible non-destructive avalanche breakdown effect in diamond. This effect has been studied as function of the concentration of impurities and temperature.

Epitaxial boron doped diamond layers were grown on (100) oriented diamond substrates by microwave plasma enhanced chemical vapour deposition (MW PECVD) in an AX5010 reactor from Seki Diamond Systems with a base pressure below $2 \times 10^{-6}$ mbar. Diamond substrates are synthetic high pressure high temperature lb Sumicrystal UP crystals from Sumitomo Electrics Hartmetall $\mathrm{GmbH}$ that have been re-polished using scaife's technique to remove subsurface damages caused by the previous polishing process. Polishing were carried out on the soft polishing direction to minimise any sub surface damages and to produce a smooth surface with a root mean square roughness $R_{R M S}<1 \mathrm{~nm}$. Substrates were cleaned in oxidizing mixture of hot sulphuric acid and potassium nitrate for $10 \mathrm{~min}$, rinsed in hot deionized water, and further cleaned in acetone and isopropyl alcohol in ultrasonic bath for 10 min each and finally air dried before loading in the CVD reactor.

Prior to the epitaxial growth, the substrates were etched in hydrogen/oxygen mixture plasma for $10 \mathrm{~min}$. Doped diamond layers (2 $\mu \mathrm{m}$ thick) were grown in a mixture of $1 \%$ of methane diluted in hydrogen at a total pressure of 100 mbar, a microwave power of $550 \mathrm{~W}$ and a substrate temperature of $1000-1100{ }^{\circ} \mathrm{C}$ using trimethylboron precursor. Epitaxial layers are smooth $\left(R_{\mathrm{RMS}} \sim 1 \mathrm{~nm}\right)$ and free of surface defects. Electrical properties of layers studied in this work were determined by Hall effect method at room temperature using a commercial Accent HL 5500PC measurement setup. Resistivity, mobility and carrier concentration are reported in Table I. Activation energy has been determined by temperature dependant measurement for temperature below $500 \mathrm{~K}$. Acceptor concentration has been estimated from reported electronic properties of boron doped CVD diamond layer by W. Gajewski et al. ${ }^{7}$. Quasi static current-voltage characteristics of boron impurity breakdown in diamond have been carried out in air and at room temperature on specially designed test devices by pulsed measurement using a Transmission-Line Pulsing (TLP) technique ${ }^{8}$. High electrical field test devices consist of two ohmic contacts separated by a boron-doped diamond bar with variable length $(d)$ from 4 to $40 \mu \mathrm{m}$. These devices and Hall bars have been prepared by a conventional reactive ion etching system (Vacutec, 1540 RIE/PE system) using sputtered aluminium mask prepared by mask less photolithography using a MicroWriter $\mathrm{ML}^{\mathrm{TM}}$ laser lithography System from Lot Oriel and wet chemical etching. Diamond layers were etched in an $\mathrm{Ar}, \mathrm{O}_{2}, \mathrm{CF}_{4}$ and $\mathrm{He}$ mixture (10:40:1:1 in volume) at a total pressure of 0.26 mbar with a $400 \mathrm{~W}$ RF power and resulting in a maximum auto polarisation bias voltage of $400-430 \mathrm{~V}$. The etching rate of the doped diamond and aluminium mask are about $50 \mathrm{~nm} / \mathrm{min}$ and less than $3 \mathrm{~nm} / \mathrm{min}$, respectively. Ohmic contacts consist of evaporated Ti/Au layers, deposited using an Edwards Auto 500 Vacuum System, annealed at $450{ }^{\circ} \mathrm{C}$ for $30 \mathrm{~min}$ in vacuum. They were also patterned by photolithography and wet chemical etching methods. The Transmission-Line Pulse generator used in this work is an Oryx Celestron 1 system that is set to deliver pulses of 100 ns width with $1 \mathrm{~ns}$ rise and fall time and a maximum repetition rate of $20 \mathrm{~Hz}$. As the pulse generator's internal resistance $(50 \Omega)$ is too low to limit and to measure the current after threshold voltage a $510 \Omega$ surface mount resistance was placed in series with tested samples. High-bandwidth current and voltage probes are used for the measurement of each pulse in the time domain with the help 
of a $6 \mathrm{GHz}$ bandwidth oscilloscope. The short duration of the pulses and the added serial resistance is intended to drastically reduce the self-heating and to achieve accurate current/voltage electrical characterisation of the samples at extremely high current density.

Fig. 1 shows the measured voltage and current pulses across the test device in series with the $510 \Omega$ resistor together with the calculated voltage pulse across the diamond device measured at room temperature. The first and last parts of the current and voltage waveforms are not to be considered. Indeed they result of the non-overlapping of the incident and reflected pulses (propagation effects). However, due to the flatness of the incident pulse, the waveforms after 20 ns and before $100 \mathrm{~ns}$ are effective images of voltage and current at the device despite two parasitic reflexions occurring at about 30 ns and $40 \mathrm{~ns}$ and that add up to the real device signal. Due to its high resistance $(R=36.8 \mathrm{k} \Omega)$ compared the setup serial resistor, the voltage drop across the diamond device nearly equals the applied voltages below $250 \mathrm{~V}$. At higher applied voltage, the current increases superlinearly (see Fig. 2a) and a relative portion of voltage across the device diminishes. At critical voltage, i.e. for an applied voltage higher that $300 \mathrm{~V}$, the current suddenly surges at a rate of

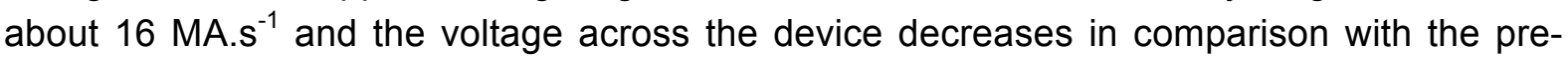
breakdown values. Fig. 2a shows the typical quasi-static characteristic current-voltage characteristic of fabricated device extracted by averaging the voltage and current waveform at the end of each TLP pulses (hashed area in Fig. 1a and Fig. 1b). This quasi-static current - voltage (I-V) characteristic exhibits five different conduction regions: 1- highly resistive ohmic region at low electric field, 2- non ohmic superlinear region at intermediate field, 3critical voltage at impurity impact ionisation avalanche, 4- transient region with negative resistance and 5- highly conductive region. Discrepancy between experimental points and theoretical ohmic I-V curve in low field is due to the insufficient measurement accuracy of the experimental setup at low currents (below $10 \mathrm{~mA}$ ). In the region (2) the (I-V) characteristic deviates from ohm's law until the breakdown.

The increase in conductivity set in first as carriers become hot and consequently carrier's thermal recombination rate is decreased until a sufficient number of carriers acquire enough energy to ionise neutral acceptors by impurity impact ionization ${ }^{6}$. The complete ionisation of dopant by avalanche effect is indicated by the current surge together with a voltage drop that results in an S-shape I-V characteristic typical of current controlled differential negative resistance ${ }^{9}$. This effect could not be observed in our previous study ${ }^{6}$ due to current limitation of the experimental setup. Differential negative resistances are associated to either contact/junction phenomena (e.g. tunnel diodes) or semiconductor bulk properties (e.g. Gunn diodes or cryosar $^{10}$ ). Similar I-V characteristic of synthetic high pressure high temperature boron doped single crystal diamond was reported by Rotner et al. ${ }^{11}$ with a breakdown field of $500 \mathrm{~V} . \mathrm{cm}^{-1}$. In their work, it was suggested that the S-shape I$\mathrm{V}$ characteristic may be caused by impurity impact ionisation avalanche despite inconsistency with the expected field. We attribute this discrepancy to thermal self-heating effect. As our observed I-V characteristic cannot be due to contact phenomena (with Nshape I-V characteristic), we ascribe its $S$ shape to impact ionization avalanche. 
There exists few reports on simulation of carriers transport properties in diamond at high electric field. Watanabe et al. have demonstrated the average energy of electron can increase well above few hundreds meV for electric field above $10^{5} \mathrm{~V} / \mathrm{cm}$ by Monte-Carlo transport simulations in diamond ${ }^{12}$. Jacoboni et al. have calculated charge transport in silicon at high electric field. Electrons and holes acquire an average energy of $\sim 400 \mathrm{meV}$ at electric field of $10^{5} \mathrm{~V} / \mathrm{cm}^{13}$. Based on these results, we estimate that carriers' energy is of the order of the boron acceptor ionization energy ${ }^{914}$ in diamond at breakdown voltage. Noticeably, the avalanche is maintained at a lower sustaining field compared to critical field. The increase by two decades of current appears consistent with full ionization of dopants considering the acceptor and carrier concentrations at room temperature. These measurements are repeatable until the filamentation of the current at highest voltages that permanently damage diamond device. The current density is extremely high (about $2 \mathrm{MA} . \mathrm{cm}^{-2}$ ) after the avalanche threshold voltage. The average power $(P=V . I)$ dissipated in the device prior to the breakdown (region 3 ) is below $3 \mathrm{~W}$ whereas it reaches about $100 \mathrm{~W}$ in region 5 , i.e. the region of complete impurity ionization. Consequently, thermal effects must be considered, especially on activation of dopants. The energy released within nanoseconds increases significantly the device's temperature as simulated using a simplified model in COMSOL multiphysics simulation software. The model is based on the devices' geometry and heat transfer equation including an electric current heat source. The electrical conductivity of diamond layer was modelled as a smoothened stepwise function of voltage to account for the sharp increase in conductivity after breakdown voltage. The simulation shows that the heat can neither be effectively dissipated through conduction into substrate, nor convection into air, nor radiation. Consequently, temperatures beyond $1000{ }^{\circ} \mathrm{C}$ in the centre of the test sample after breakdown have been computed. Note that the simulation does not account for the possible local conductivity of the surrounding substrate that might be induced by carriers' injection from the bar. This would presumably delocalise the thermal effects and, hence, lower the temperature. This temperature is enough to ionize all boron acceptors; however, as shown in our previous study ${ }^{6}$, measured I-V characteristics clearly show that the super linear increase of the current, due to carriers multiplication, occurs at same power dissipation level for which no current multiplication is observed at higher temperature. This result demonstrates that the observed initial carriers' multiplication is due to electric effect and cannot be due to thermal effect.

The critical avalanche voltage has been determined as the highest voltage prior to the I-V characteristic discontinuity. Determined critical voltages are reported in Fig. $2 b$ as function of inter-electrode gap and carrier concentration. All measured values are below Paschens' laws in air in standard temperature and pressure conditions as well as measured air breakdown on un-doped diamond layers (not shown). These results demonstrate that the observed breakdown is a diamond-bulk electrical phenomenon and not an inter-electrode arcing in air. This is further confirmed by the linearity of breakdown voltage with the inter electrode gap which clearly indicates an electric field dependent phenomenon. The breakdown voltage increases as acceptor concentration decreases (see Table I) which is opposite to classic work $^{15}$. Indeed concentration of majority and minority impurities, i.e. compensation, are the main parameters that determine the breakdown field. In germanium, breakdown field is known to increase with dopant concentration and compensation as for dopant concentration between $6.1 \times 10^{13}$ and $3.1 \times 10^{17} \mathrm{~cm}^{-3}$. However, our results are consistent with Sankin et 
al. work on silicon carbide who reported an increase of impurity impact ionisation breakdown field with decreasing aluminium acceptor concentration ${ }^{5}$. This behaviour is attributed to the substantial decrease of activation energy at high dopant concentration $\left(\geq 10^{19} \mathrm{~cm}^{-3}\right)^{16,17}$.

Quasi-static current-voltage characteristics of sample 1 (see Table I), with interelectrodes gap of $22.4 \mu \mathrm{m}$ and bare width of $12.1 \mu \mathrm{m}$, measured at substrate's temperature between 15 and $100{ }^{\circ} \mathrm{C}$ are shown on Fig. 3a. Temperature rise reduces devices resistance, resistivity and most significantly the critical voltage and field. Noticeably, all current-voltage characteristics in the high conduction region converge to the same I-V characteristics with a characteristic slope of $4.5 \mathrm{~mA} / \mathrm{V}$, i.e. an equivalent resistivity of $24 \mathrm{~m} \Omega . \mathrm{cm}$ that is in agreement with full ionisation of neutral impurities. The variation of critical field with temperature is consistent with impurity impact ionisation avalanche ${ }^{18}$. We experimentally observe a $\mathrm{T}^{-}$- temperature dependence of the critical field with $\alpha=1.3$ (see Fig. 3b). Amazingly, this result is similar to the temperature dependence of critical field results reported by Koenig et al. ${ }^{18}$ in doped germanium at low temperature.

In summary, we have investigated electrical properties of $p$ type semiconducting diamond in high electric field. Quasi static current-voltage characteristic of CVD doped (100) diamond epitaxial layers with variable boron concentration have been measured by Transmission-Line Pulse method between 15 and $100^{\circ} \mathrm{C}$. All studied samples exhibit an Sshape current-voltage with a highly resistive ohmic region at low electrical fields and a highly conductive region at high electric fields that are separated by a transient negative resistance region. This behaviour is attributed to impurity impact ionisation avalanche and consequent full ionization of neutral boron impurity. Determined values of avalanche critical fields are consistent with those expected from theoretical estimations and with our previous report ${ }^{6}$. Like for silicon carbide ${ }^{5}$, their increase with decreasing acceptor concentration is attributed to the reduction of ionisation energy of dopant in the range of studied boron concentration while its temperature dependence is consistent with classic impurity impact ionisation. Still more detailed studies must be carried out to completely describe electrical conduction mechanisms in doped diamond in high electrical fields.

Acknowledgements: Financial support from the following sources are gratefully acknowledged: Academy of Sciences of the Czech Republic, Czech Science Foundation (GACR) Grant ID: GAČR 13-31783S, the French-Czech Project PHC BARRANDE 2016 35785SC "High Electric Field Properties of Doped Diamond and Applications" and J.E. Purkyně fellowship awarded to V. Mortet by Academy of Sciences of the Czech Republic.

\section{References:}

${ }^{1}$ A.K. Jonscker, Proc IEEE 52, 1092, 1964.

${ }^{2}$ D.L. Dreifus (1995) Diamond: electronic properties and applications. L. S. Pan and D. R. Kania (Eds.) Dordrecht/Boston/London: Springer Science+Bussiness Media, LLC.

${ }^{3}$ N. Sclar and E. Burstein, J. Phys. Chem. Solids 2, 1, 1957.

${ }^{4}$ N. Kawamura, J. Phys. Chem. Solids 27, 919, 1966. 
5 V.I. Sankin, A. M. Monakhov, P. P. Shkrebiy, P.L. Abramov, N.I. Sablina and N.S. Averkiev, Appl. Phys. Lett. 97, 262118, 2010.

${ }^{6}$ V. Mortet, A. Soltani, Appl. Phys. Lett. 99, 202105, 2011.

${ }^{7}$ W. Gajewski, P. Achatz, O. A. Williams, K. Haenen, E. Bustarret, M. Stutzmann, and J. A. Garrido, Phys. Rev. B 79, 045206, 2009.

${ }^{8}$ T. J. Maloney,N. Khurana, Proc. EOS/ESD symp. 49, 1985.

${ }^{9}$ B. K. Ridley, Proc. Phys. Soc. 82, 954, 1963.

${ }^{10}$ T. Kurosawa Japan. J. Appl. Phys. 1 (1962) 365

${ }^{11}$ Y. M. Rotner, V. A. Presnov, N. N. Golembievskii, L.F. Litovchenko, S.M. Rotner and V.A. Laptev, Sov. Phys. Semicond 8, 1293, 1975.

${ }^{12}$ T. Watanabe T. Teraji, T. Ito, Y. Kamakura, K. Taniguchi, J. Appl. Phys. 95, 4866, 2004.

${ }^{13}$ C. Jacoboni, C. Canali, G. Ottaviani A. Alberigi Quaranta, Solid-states, 20, 77,177.

14 J. Parisi, U. Rau, J. Peinke, K.M. Mayer, Z. Phys. B - Condensed Matter 72 ,225, 1998.

${ }^{15}$ F. Karel, J. Oswald, J. Pastrnak, O. Petricek, Semicon. Sci. Tech. 7, 203, 1992.

16 P. Achatz, J. Pernot, C. Marcenat, J. Kacmarcik, G. Ferro, E. Bustarret, Appl. Phys. Let. 92, 072103, 2008.

17 J.P. Lagrange, A. Deneuville, E. Gheeraert, Diam. Relat. Mater. 7, 1390, 1998.

${ }^{18}$ S.H. Koenig and G.R. Gunther-Mohr, J. Phys. Chem. Solids 2, 268, 1957.

${ }^{19}$ R.M. Schaffer "Electrophotography" John Wiley and Sons, 1975, p.517 
TABLE I.

\begin{tabular}{r|cccc}
\hline \hline Parameter & Sample 1 & Sample 2 & Sample 3 & Units \\
\hline Carrier concentration $([\mathrm{p}])$ & $1.7 \times 10^{17}$ & $4.1 \times 10^{16}$ & $4.0 \times 10^{15}$ & $\mathrm{~cm}^{-3}$ \\
Estimated acceptor concentration & $3 \times 10^{19}$ & $2 \times 10^{19}$ & $1 \times 10^{19}$ & $\mathrm{~cm}^{-3}$ \\
Carrier mobility $(\mu)$ & 9.3 & 21.5 & 286 & $\mathrm{~cm}^{2} . \mathrm{V}^{-1} \cdot \mathrm{s}^{-1}$ \\
Resistivity $(\rho)$ & 3.8 & 7.3 & 5.5 & $\Omega . \mathrm{cm}$ \\
Activation energy $\left(\mathrm{E}_{\mathrm{A}}\right)$ & 210 & 230 & 265 & $\mathrm{meV}$ \\
Breakdown field & 128 & 167 & 214 & $\mathrm{kV} . \mathrm{cm}^{-1}$ \\
\hline \hline
\end{tabular}

Table I: Electrical properties of studied boron doped diamond 

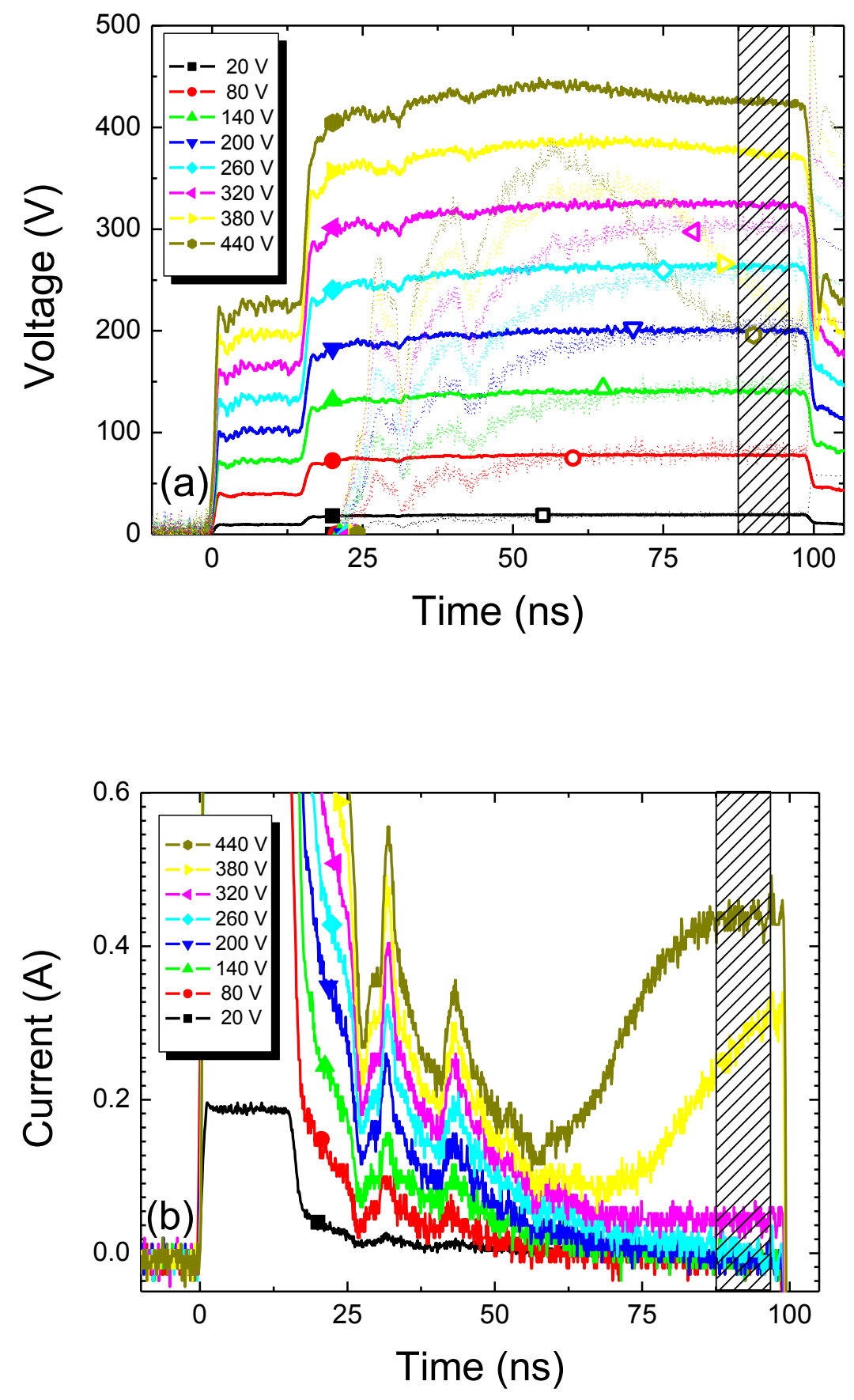

FIG. 1. (a) Measured voltage pulses across the diamond test device in series with the 510 $\Omega$ resistor (full lines) and calculated voltage pulses across the test device (dotted lines); (b) Measured current pulses (003-L62- L=22.4 $\mu \mathrm{m} / \mathrm{w}=12 \mu \mathrm{m} ; \mathrm{R}=36.8 \mathrm{k} \Omega$ ). 

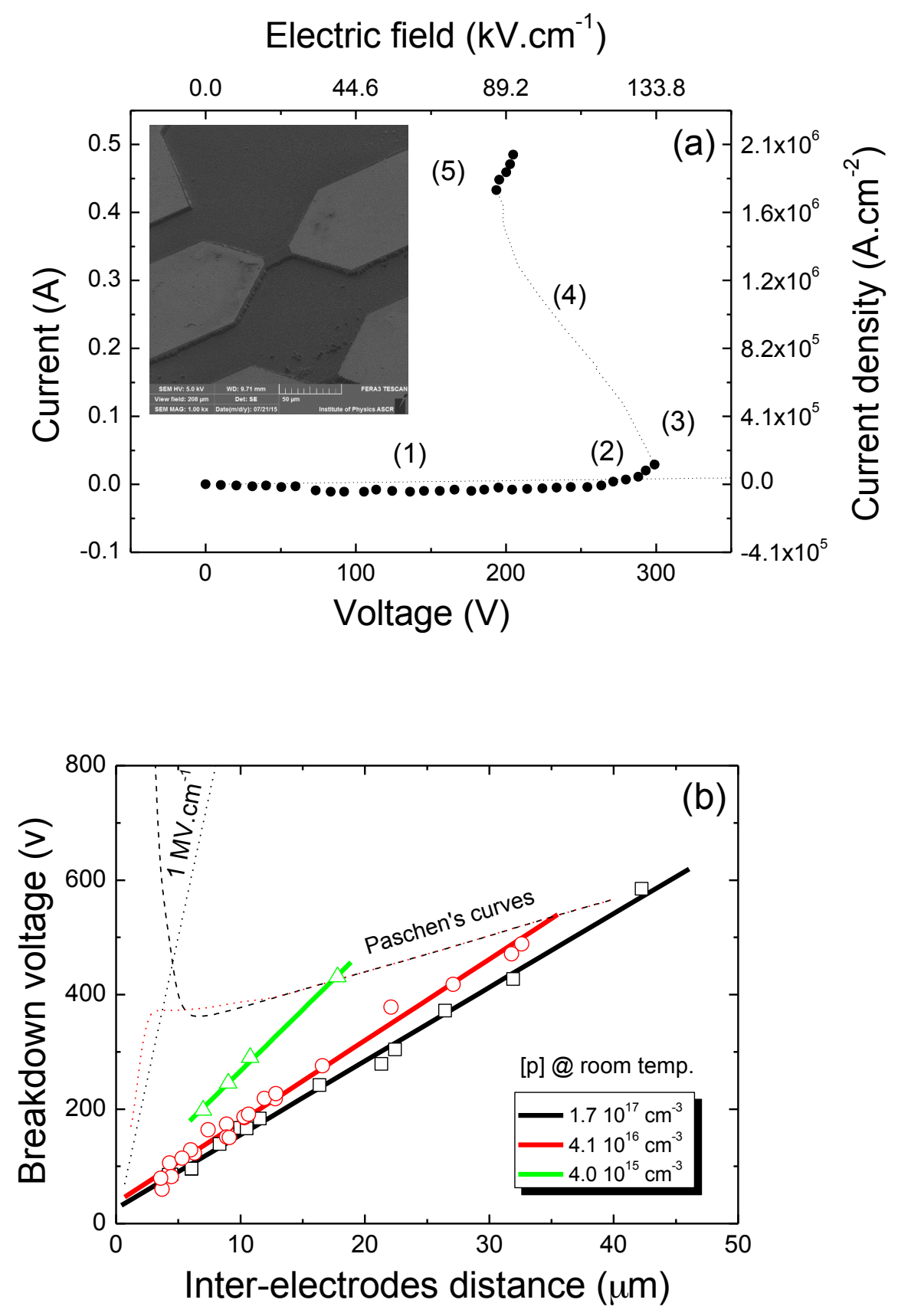

FIG. 2. (a) Quasi-static I-V characteristic of a $22.4 \mu \mathrm{m}$ long boron doped diamond device with $[\mathrm{p}]^{300 \mathrm{~K}}=1.7 \times 10^{17} \mathrm{~cm}^{-3}$. Theoretical linear ohmic I-V characteristic with $\mathrm{R}=36.8 \mathrm{k} \Omega$ resistance is plotted for comparison (dotted line) (inset: Scanning electron microscopy images of high electric field test devices); (b) Experimental breakdown voltage (dots) as function of the inter-electrodes distance of sample 1-3 with different acceptor concentration with their linear fit (line). Breakdown voltage characteristics in air and at atmospheric pressure as function of a gap, i.e. Paschen's (dashed line) and modified Paschen's (dotted line $)^{19}$ curves are shown for comparison. Lower limit of undoped diamond breakdown voltage of (i.e. with breakdown field of $1 \mathrm{MV} . \mathrm{cm}^{-1}$ ) is also reported for comparison. 

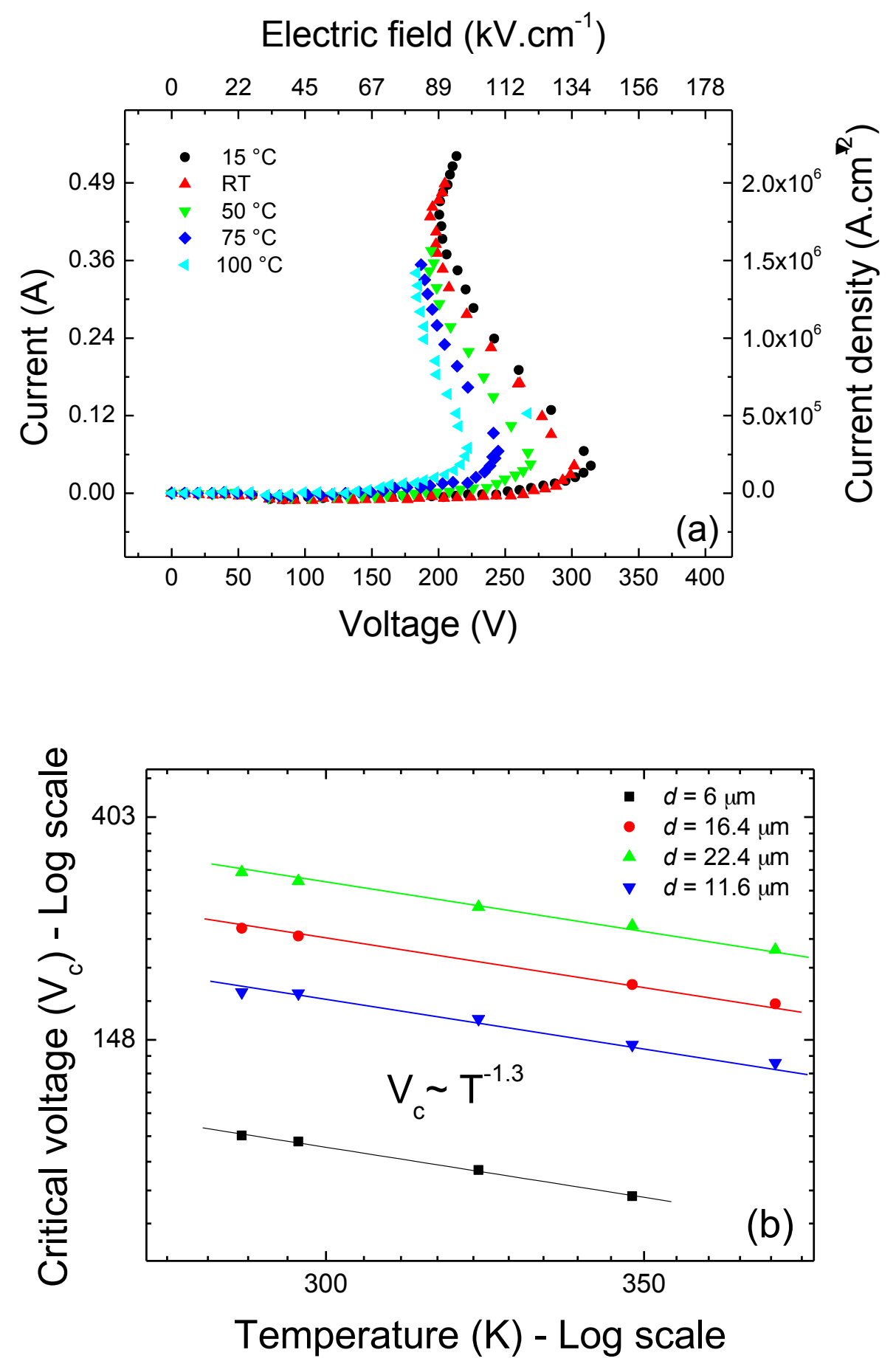

FIG. 3. (a) Quasi-static I-V characteristic (electrodes' gap: $22.4 \mu \mathrm{m}$ ) for different temperature; (b) variation of breakdown voltage as function of temperature for different interelectrodes distances $(d)$ of sample 1. 